\title{
OR UNA GOBERNANZA EN TECNOLOGÍA MENOS INGENUA
}

Gloria Baigorrotegui B ${ }^{1}$

\author{
Centro de Integración Ingeniería y Sociedad. \\ Universidad de Santiago de Chile \\ Correo Electrónico: gbaigorr@1auca.usach.cl
}

Resumen: En el siguiente trabajo continuaré ${ }^{2}$ la crítica desarrollada a las perspectivas constructivistas en tecnología, en esta ocasión me centraré en los procesos de gobernanza en tecnología. Para esto introduciré los enfoques de la Construcción Social de la Tecnología (CST) (Bijker, 1995; Pinch y Bijker, 1984) y la Teoría del Actor Red (TAR) (Latour, 1987, 1983; Callon, 1987; Law, 1987, 1991), los cuales influyen en la perspectiva de la Evaluación Constructiva de la Tecnología (ECT) (Rip, 1994, 2002; Rip et al, 1995), que trabaja particularmente en torno a la formulación de políticas públicas en tecnología.

En segundo término, expondré el enfoque de los Sistemas Socio-Técnicos de Gran Escala (SSTGE) (Hughes, 1987, 1983, 1996), para ubicar la gestión de conflictos en instituciones con estilos poco tolerantes a las resistencias de su entorno. Añadidamente, me referiré a los gobiernos más autoritarios y, a partir de esta situación, incorporaré los planteamientos sobre la gestión de la intensidad hostil provenientes de los grupos resistentes y afectados (Galtung, 1971). Para finalizar entregaré algunos delineamientos a favor de la gobernanza en ciencia y tecnología activada desde los grupos resistentes.

\footnotetext{
${ }^{1}$ Doctora en Estudios Sociales de la Tecnología de la Universidad del País Vasco/Euskal Herriko Unibersitatea.

${ }^{2}$ El trabajo previo es mi tesis doctoral en la cual desarrollé una crítica hacia los enfoques constructivistas desde la perspectiva de las teorías de conflictos (Baigorrotegui, 2008).

Fecha de recepción: 4 de mayo de 2009

Fecha de aceptación: 26 de junio de 2009
}

Palabras Clave: gobernanza en tecnología configuración social de la tecnología - gestión socio-técnica de conflictos - movilizaciones ciudadanas.

Abstract: In the following work I continue the criticism developed to the perspectives constructivists in technology, in this occasion this critic it's centred in the governance of technology processes. For this I introduce Social Construction of Technology (SCOT) (Bijker, 1995; Pinch y Bijker, 1984) and the Actor Network Theory (ANT) (Latour, 1987, 1983; Callon, 1987; Law, 1987; 1991) approaches, which they influence in the Constructivistic of Technology Assesment (CTA) (Rip, 1994, 2002; Rip et al, 1995) perspective, that works particularly around the formulation of public policy in technology.

In second term, I expose Large Socio-technical System (LST) (Hughes, 1987, 1983, 1996) approach, it serves me to locate the management of conflicts in institutions characterized for few tolerant styles in front of environmental resistances. Besides, I refer to the most authoritarian governments and, from this situation I incorporate the approaches on the management of the hostile intensity originating from the resistant groups and affected (Galtung, 1971). To finalize I deliver some delineations in favor of the gobernance in science and technology activated since the resistant groups..

Key words: governance in technology - social shaping of technology - management of socio-technical conflicts citizenship movements. 


\section{Gobernanza en tecnología}

\section{y sus fuentes constructivistas}

La gobernanza, dentro de sus acepciones constructivistas, comprende las prácticas políticas como procesos interactivos y reflexivos, es decir, como procesos de coconstrucción (Rip, 1994, 2002).

Es por esto que los temas en torno a la gobernanza se ocupan no sólo del rol de las personas ubicadas en sus oficinas, administraciones y organizaciones para definir y delimitar sus identidades, sino que además se centran en los procesos que van constituyendo aquellas identidades en acción. Smith \& Stirling destacan las relaciones diversas que dan forma a los procesos científico-tecnológicos, en este sentido, ambos autores centran su trabajo en aclarar, por ejemplo: ¿cómo se dirigen las estrategias de política científico tecnológica?, ¿cómo son definidos los recursos para la salud y el medioambiente? y ¿cómo son distribuidos los daños medioambientales? Después de indagar acerca de estos cuestionamientos, Smith \& Stirling describen y explican los procesos políticos como relaciones sociotécnicas que configuran $-\mathrm{y}$ son configuradas por- las interacciones entre personas, tecnologías y medioambiente (Smith \& Stirling, 2006 p. 1-2).

Aquí quiero aclarar que la influencia de las perspectivas constructivistas en tecnología impregna las discusiones en torno al poder y por ello se refieren a la gobernanza como modalidad apropiada para llevar adelante objetivos de sostenibilidad y participación ciudadana. Mi preocupación se relaciona con la escasa cautela en esta interpretación, especialmente cuando las estrategias en tecnología corresponden a un porcentaje de aquellas utilizadas en política. A continuación introduciré brevemente los enfoques de la Construcción Social de la Tecnología (CST) y la Teoría del Actor-Red (TAR) para aclarar en qué contexto comprendo esta extrapolación y mi crítica sobre su ingenuidad.
La tecnología entendida como "construcción social" desciende del interés inicial por comprender la construcción de hechos científicos en controversia, interés liderado por el Programa Empírico del Relativismo (Bloor, 1976) ${ }^{3}$, el cual con posterioridad cedería su entramado teórico para que autores como Pinch \& Bijker presentaran el programa de la Construcción Social de la Tecnología (CST) (Pinch \& Bijker, 1984) para explicar los artefactos y sistemas tecnológicos como entidades intrínsecamente sociales.

La configuración social de la tecnología es, según estos autores, posible de rastrear a partir de una labor del analista a cargo de deconstruir los diseños tecnológicos: i) se rastrean las distintas interpretaciones que un diseño ha tenido en su historia, lideradas por su correspondiente Grupo Social Relevante (GSR)4, ii) se analizan los mecanismos por medio de los cuales determinadas interpretaciones propias de un GSR- logran posicionarse en la lucha sobre otras interpretaciones, iii) se realizan generalizaciones para conectar los mecanismos encontrados -responsables de estabilizar el diseño particular- con un marco de interpretación envolvente de esa tecnología.

Bajo esta óptica, el analista deconstructivista saca a la luz aquellas interpretaciones que en el discurso oficial -eficientista y tecnócrata de los artefactos- quedaron invisibles. En este sentido, desde CST se acentúa la multiplicidad de vías por las que se constituyen los diseños tecnológicos, y con ello, se destaca en el análisis que otros diseños también pudieron ser los vencedores. El grupo social relevante vencedor junto a su interpretación

${ }^{3}$ El Programa Fuerte de la escuela de Edimburgo a mediados de los años setentas, formado por David Bloor, Barry Barnes, David Edge y Donald MacKenzie, declaró los siguientes principios base para el estudio de la ciencia: i) Causalidad: los estudios sociales de la ciencia deberían explicar no sólo los estados de conocimiento sino también las creencias sociales; ii) Imparcialidad: Los estudios sociales del conocimiento deberían ser imparciales en torno a los juicios y valoraciones respecto a la ciencia iii) Simetría: el mismo tipo entramado de causas servirían para dictaminar entre conocimiento falible o verídico tanto en ciencia como en sociedad; iv) Reflexividad: lo que se aplique para la ciencia debe ser capaz de aplicarse para los estudios sociales de ella (Bloor, 1976).

${ }^{4}$ La noción de Grupo Social Relevante GSR apunta hacia una diversidad de formas organizativas e informales, pero se prioriza en ellas el hecho de compartir un grupo de significados específicos asignados al artefacto en cuestión (Pinch \& Bijker, 1984, pág. 414) 
triunfante no es evidente, ni será el ganador eterno, dado que emerge de una red con lógicas entrecruzadas y eventos contingentes, y además siempre estarán listos otros grupos con otros marcos tecnológicos para amenazar sus posiciones de poder.

Esta visión dinámica de las tensiones de los diseños tecnológicos liderados por grupos sociales relevantes y sus correspondientes interpretaciones no coincide con el programa conocido como la Teoría-Actor-Red (TAR), el cual califiqué como construccionalistas.

TAR propone que los artefactos, materiales, discursos y sistemas tecnológicos sean entendidos como resultado de la traducción de intereses realizadas por Actores-Red, o sea, actores que no son de carne y hueso exclusivamente, sino híbridos humano-artefactuales. Esta condición lo diferencia del constructivismo social y obedece a su adscripción al principio de simetría generalizada ${ }^{6}$. Esta ampliación del principio tiene el objeto de evitar que los analistas constructivistas rastreen únicamente aquellas interpretaciones propias de personas o grupos en torno a un artefacto o sistema técnico, y elijan integrar simétricamente -con igual importancia- a las abstracciones, representaciones, configuraciones materiales y las personas o grupos que comparten significados en torno

\footnotetext{
${ }^{5}$ Este calificativo obedece a la distinción que Hacking realiza de los constructivismos, en este sentido, la teoría-actor-red sería un enfoque más cercano a lo que Hacking define como construccionalismo. Desde esta perspectiva las representaciones numéricas, abstracciones, mapas, materiales colaboran de forma importante en la constitución de un artefacto. Con esto quieren dejar en evidencia que los aspectos materiales de la construcción no deberían subyugarse al estudio exclusivo de interpretaciones, en este sentido, juegos puramente discursivos, proveniente de los grupos sociales relevantes (Hacking, 2001).

${ }^{6} \mathrm{El}$ principio de simetría generalizada proviene del principio de simetría, heredero del programa empírico del relativismo. La causa de esta adjetivación nueva del principio de simetría, ahora generalizada, tiene el ánimo de entregar una interpretación más radical: explicar no sólo las perspectivas y argumentos discursivos en un enfrentamiento en ciencia y tecnología, sino también las impurezas que las constituyen, para esto es necesario mantener un mismo registro explicativo cuando el analista transita entre argumentos técnicos y argumentos sociales en disputa (Callon, 1995: 261-2).
}

a un artefacto. Con esto, TAR reivindica el rol de los artefactos y sus maquinaciones de poder en la lucha por transformarse "puntos de paso obligado" dentro de la red de "ensamblajes heterogéneos". Así la generalización de la simetría es radical porque no requiere para su aplicación que sean distinguidos las interpretaciones de los GSR acerca de un artefacto y el artefacto en sí.

Law demuestra sus desavenencias con Bijker cuando alerta sobre la supremacía otorgada en SCOT a las interpretaciones de los grupos sociales relevantes, mientras Bijker le responde que ésta no sería tal dado que productos y marcos tecnológicos también influyen en estos grupos, interpretativa y estructuralmente (Law, 1987).

No obstante, tanto constructivistas sociales (CST) como construccionalistas (TAR) concuerdan en que la tecnología no es más heredera de una lógica interna independiente de aspectos sociales;

ambos enfoques reconocen que sin estos últimos aspectos la tecnología sería incapaz de librar batallas de poder, debido a que sería nada más que basura inerte.

La respuesta a la pregunta acerca de cómo los aspectos sociales van configurando $-\mathrm{y}$ configurándosetecnológicamente es controvertida: en CST se trataría de luchas por estabilizar la interpretación privilegiada tratando de integrarse (en), acercarse (a) marcos tecnológicos envolventes; mientras que en TAR las luchas por tornarse "punto de paso obligado" requieren que el actor-red simplifique y yuxtaponga. Lo primero es un impulso práctico en el cual los Actores-Red limitan sus asociaciones a un set de entidades con características definidas, es decir, sería una reducción del mundo para lograr asociaciones posibles. Todas estas simplificaciones existirían en contextos, es decir, en yuxtaposición a otras entidades con las cuales están ligadas y a través de las cuales los actores definen las condiciones de operación de sus diseños. La yuxtaposición entrega coherencia, consistencia y estructura a las relaciones entre componentes. 
Aunque TAR y CST concuerden en destronar una idea lineal y artefactual de la tecnología, ambos discrepan en cómo interpretar los aspectos más atrincherados y estabilizados, y por ello ambos enfoques enfrentan críticas.

Para Collins \& Yearley, la reivindicación de los artefactos en TAR está al límite de transformarse en una postura determinista de la tecnología, postura contra la que tanto tiempo se ha luchado en los estudios sociológicos del conocimiento científico (Collins \& Yearley, 1992); por su parte, Brown \& Lee proponen descubrir el continente no descubierto por TAR, reconociendo ubicuidad desde la perspectiva todo-abarcante del Actor-Red (Brown \& Lee, 1998). Asimismo, Jæger critica a CST por sus deficiencias para interpretar macro-estructuras y con ello lo infructuoso de realizar análisis comparados (Jæger, 2001); mientras que Hård se preocupa porque en CST se considere la clausura de un diseño por parte de un GSR como sinónimo de consenso social (Hård, 1993). Finalmente es Winner quien sostiene que las corrientes constructivistas de la tecnología, en general, describen la tecnología de una forma inmune a contagios ideológicos y partidistas, tanto así que resultan siendo análisis conservadores y reiterativos hasta la saciedad sobre la idea que la tecnología es construida socialmente (Winner, 1993).

Retomando estas críticas, autores como Arie Rip se han alejado de este halo de neutralidad que pareciera rondar por los pasillos constructivistas ${ }^{7}$ y han decidido abrir sus puertas y explicitar que la naturaleza de sus contribuciones en tecnología, son del tipo de "asesorías profesionales".

\footnotetext{
${ }^{7}$ Esta iniciativa la prosigue también el programa de la Configuración Social de la Tecnología, más conocido por su acrónimo inglés-Social Shaping of Technology- (SST). Como señaló Williams \& Edge (1991) esta "iglesia heterodoxa" de los estudios sociales de la tecnología se preocupa por los procesos más que los productos científico tecnológicos; bajo este entendido, una tecnología será valorada como exitosa, siempre y cuando, incluya procesos flexibles e inclusivos lo antes posible, por sobre aquellas tecnologías a cargo de gestionar procesos controlados completamente. Este criterio de valoración se conecta así con las propuestas políticas sobre la inclusión de los ciudadanos en las decisiones políticas en ciencia y tecnología que les interesa, preocupa, afecta directa o indirectamente. Sin embargo, esta ligazón entre las decisiones científico tecnológicas y los objetivos de inclusión y participación social ha sido más discursiva que concretada.
}

Este es el caso del enfoque de la Evaluación Constructiva de la Tecnología (ECT), enfoque gracias al cual los analistas se reconocen como asesores y no sólo como personas solitarias tras sus escritorios y publicaciones. Para Arie Rip la tecnología es gobernanza, en otras palabras: el hecho que los aspectos sociales construyan y sean construidos por los aspectos materiales, es decir, sean co-construidos socio-materialmente corrobora los términos en que se entiende la "gobernanza en sí misma".

Para Rip si las personas se concentrasen más en los procesos que en los productos tecnológicos, comprenderían en qué consiste la co-construcción, es decir, la coexistencia participativa de agencias de distinta índole y esto, a su vez, permitiría a todos aprender con ello. Desde esta perspectiva constructivista, una política tecnológica apropiada es aquella abocada al diseño de procesos flexibles y participativos desde sus inicios, antes que los productos y servicios salgan a la luz. Rip con esto reclama que las políticas científico tecnológicas se hayan concentrado más en la gestión de conflictos y no tanto en el aprendizaje social (Rip et al, 1995: 350).

Hasta aquí mi exposición de los enfoques constructivistas de la tecnología CST y TAR y su traducción en términos de gobernanza bajo la perspectiva de la ECT. Esta exposición ha tenido el objeto de presentar las principales preocupaciones de estas aproximaciones y sus correspondientes críticas. A partir de ahora desarrollaré la mía en torno a la ingenuidad mostrada por las interpretaciones constructivistas en tecnología referidas a la gobernanza. Para exponer mi defensa presentaré la aproximación de los sistemas sociotécnicos de gran escala (Hughes, 1983) y a partir de ella, me referiré al interés de gobernar los conflictos al interior de sistemas.

\section{Gobernabilidad en los sistemas socio-técnicos globales}

Hughes propone el enfoque histórico de los Sistemas SocioTécnicos de Gran Escala (SSTGE) concentrado en explicar 
las dinámicas de sistemas tecnológicos determinados en distintas etapas evolutivas (Hughes, 1983, 1987). El surgimiento de los sistemas, entendidos como todos sinérgicos, no es ajeno a la noción de luchas y oportunidades contingentes, las cuales logran resolverse y encontrarse respectivamente gracias a la labor de sus constructores particulares: los constructores de sistemas. Estos actores serían los encargados de resolver ingeniosamente los problemas clave -reverse salients- en el momento apropiado para salvar al sistema del estancamiento o la extinción, y así poder seguir ampliándolo, estabilizándolo hasta llegar a su momentum. En esta etapa, el sistema sociotécnico parecería volverse autónomo; lo que significaría que las instituciones resultarían siendo sus aliadas, los entramados de regulación y políticos se interesarían en su perpetuación, las personas usuarias se mostrarían confiadas en su funcionamiento, entre otras.

En SSTGE, eso sí, las personas no son igual de importantes que los artefactos. Los grados de libertad en las primeras son mayores que en los segundos, es por esto que desde una perspectiva óntica de los sistemas: humanos y artefactos no son simétricos. Ahora bien, el sistema concebido como un "todo sinérgico" obedece a su identidad y sentido; cada acción se supone coherente y colineada, es por esto que existe jerarquía en las acciones, así los desacuerdos y tensiones al interior de los sistemas no podrían sobrepasar un umbral de hostilidad, el cual resultase ingobernable. La coherencia de la identidad del sistema requiere de una gestión cuidada de controversias y conflictos en su seno. De este modo, las configuraciones socio-materiales no pueden desestabilizarse por completo porque con ello peligra el sentido y la misión del sistema.

La gestión de conflictos en el sistema se relaciona con los esfuerzos para definir y redefinir la identidad, la misión y los sueños venideros que sus miembros pueden compartir, en extremo podría considerarse como una especie de adoctrinamiento sistémico, sin embargo la pertenencia en un sistema puede ser participativa, negociada, heredada 0 impuesta.
Aun cuando, en un sistema la noción de control es necesaria para explicar la tendencia hacia su crecimiento, también es cierto que ésta resulta insuficiente para comprender su supervivencia.

Esto quiere decir que no toda materialización tecnológica tiene la tendencia a crecer tan controlada y planificadamente como, por ejemplo, lo hacen sistemas de distribución eléctrica. Sin embargo, cuan infranqueable sean los límites de un sistema dependerá de la relación entre sus miembros, la configuración material de la tecnología y dependerá también de las posibilidades que los directivos ofrezcan a sus integrantes para participar, deliberar, negociar y cuestionar los objetivos y visiones futuras. Asimismo, no se podría descartar del análisis que los diseños tecnológicos -especialmente los de gran escala- surgen germinados por visiones paradigmáticas, las que a su vez actúan como envolventes de significado y prefiguran su entorno como materializaciones concretas. En palabras de Hughes, los sistemas tecnológicos específicos actuarían como "suaves bestias" comportándose como sus diseñadores las crearon, dentro de límites de control, muchas veces, de manera irreversible. Para esto, alguien se encarga de llevar las riendas del desenvolvimiento de "las bestias", es decir, una persona 0 un grupo directivo, una agencia capaz de liderar los cambios y obstaculizar las desviaciones al interior o en el entorno inmediato del sistema. El estilo con el cual los directivos proyecten sus asociaciones y aspiren a ejercer el control de sus proyectos dará el cariz participativo 0 tolerante de sus intervenciones tecnológicas en sociedad, la cual puede no ser conciliadora necesariamente.

Con esto quiero aclarar que no todas las directivas de los sistemas tecnológicos se caracterizan por la inteligencia y el pacifismo con el cual dirimen sus inconvenientes; una prueba de ello es el auge de las certificaciones en

\footnotetext{
${ }^{8}$ Esto lo menciono como crítica a Hughes, quien reconoce en los constructores de sistemas una capacidad innata para resolver y activar mecanismos pacíficos e inteligentes con tal de que el sistema perviva (Hughes, 1987).
} 
responsabilidad social empresarial. Este mismo cariz relativo a la falta de amigabilidad puede reconocerse en los directivos del Estado, en los gobiernos.

El acto de gobernar y sus gobernantes con tal de facilitar la acción colectiva en el seno del Estado-nación han sido embestidos históricamente de la facultad de acallar los conflictos. De este modo, los grupos contestatarios, la oposición y las acciones de resistencia de los grupos no estatales han sido interpretadas como desventajas para los objetivos comunes (Stoker, 1998). Para Martin los espacios de deliberación y participación son escasos para los grupos de resistencia, debido a que, según él, en gran medida éstas dependerán de los estilos de gobierno, y cuán tolerantes sean las élites con sus resistentes. Como es sabido; si un gobierno es elegido en las urnas, esto no garantiza que su ejercicio sea democrático, inclusivo, deliberativo 0 negociador. Martin con esto destaca cuán simultáneos y complejos son los sistemas políticos y cuán injusta e indiscriminadamente pueden utilizarse las fuerzas públicas de orden contra los subversivos. Panfletos y comunicados de prensa podrían ser leídos como actos de sedición en regímenes autoritarios; mientras que las mismas acciones de los trabajadores, en los sistemas representativos podrían tener respuestas represivas por parte de los ejecutivos de la empresa (Martin, 2006: § 2.3).

Por un lado, las "maquinaciones maquiavélicas" de un líder, constructor de sistemas o un Actor-Red no están libres de detonar, facilitar, e incluso promover indirectamente espacios inundados de violencia, falta de amigabilidad, y contradicción en aumento (Galtung, 1971); y por otro lado, los gobiernos no necesariamente interpretan los descontentos en su seno como espacios de posibilidad para el desenvolvimiento democrático de su Estado.

Dicho esto, los desencuentros hostiles no son actos fáciles de prever, porque son subjetivos, altamente contingentes, difíciles de poder generalizar; aunque tampoco surgen de la nada, del vacío, debido a que están alimentados y sedimentados bajo un cúmulo de historias de encuentros y desencuentros pasados entre los oponentes. Galtung con respecto a esto propone que las prácticas conflictivas son dinamizadas gracias a la interacción sinérgica de tres aspectos y dentro de ellos sólo uno sería el manifiesto y visible: el comportamiento hostil, mientras que los otros dos serían aspectos teóricos e inferidos: las contradicciones y las actitudes poco amigables entre los oponentes?.

Es por esto que no me parece razonable aceptar en los análisis constructivistas de la tecnología -y mas aún cuando se refieren a la gobernanza en sí- su exclusiva atención al punto de vista de las redes de emprendimiento de un proyecto tecnológico, la mayoría de las veces exitoso, y su olvido persistente ante la perspectiva de quienes activan el descontento, la preocupación, la duda ante determinadas prácticas tecnológicas. Incluso, es más, son estas mismas agencias, preocupadas, las que propagan la chispa capaz de encender iniciativas distintas, algunas veces posibles de concretar en la forma de contra-proyectos. Aunque pueda parecer obvio: la gobernanza también la constituyen las acciones de protesta, los actos de denuncia, los proyectos opacados, las contra-iniciativas marginadas.

Una de las razones por la cual denomino las perspectivas constructivistas ingenuas es la parcialidad con la cual sus explicaciones socio-técnicas pretenden igualarse a prácticas de gobernanza en sí. Esto -creo-desalienta el ánimo de sus lectores menos ingenuos y reduce su incuestionable poder explicativo en lo que a iniciativas socio-técnicas se trata, porque las desnudan de contexto. No me parece apropiado presentar sólo el lado posibilista de las intervenciones tecnológicas e invisibilizar en el análisis las estrategias de los adversarios, quienes en el caso de aparecer resultan ser competidores ante los cuales es mejor adelantarse para no perder el interés de los propios. Siguiendo a Galtung, las expresiones visibles, materiales de la tecnología como resultado de conflictos son la punta del iceberg conflictivo, y no sólo se constituyen de posibilidades para unos, sino que también de intolerancias y contradicciones para otros.

${ }^{9}$ Es conocido en las teorías sobre la gestión de conflictos como el triángulo conflictivo de Galtung (Galtung, 1971). 


\section{Gestión socio-técnica de conflictos}

Si bien Latour ha descrito de forma astuta cómo los artefactos generan política por otros medios, también es cierto que la práctica tecnología es generada por - y generadora de- distintos tipos de luchas, conflictos y polémicas, muchas de estas últimas, imposibles de librarse directa 0 indirectamente de dinámicas conflictivas en escalada de pasión hostil. La escalada o espiral conflictiva hace referencia a la tendencia bi-direccional en la dinámica conflictiva, una ascendente y otra descendente: "Llamamos escalada o escalamiento a un movimiento hacia niveles de mayor intensidad conflictiva de cualquiera de los actores. Inversamente, la desescalada o desescalamiento es un desplazamiento hacia niveles más bajos de intensidad" (Entelman, 2002: 177).

Los gradientes de esta intensidad conflictiva se relacionan con la modificación en los aspectos actitudinales y comportamentales de la dinámica conflictiva. La escalada conflictiva puja por generar estados de excepción, la cual no es previsible por una ley, aunque sí existe una probabilidad mayor de que ésta aparezca cuando ya no es posible discutir las incompatibilidades ni tampoco acordar procedimientos o protocolos. Así, el recurso a la escalada es impredecible y requiere al menos considerar que el grado de antagonismos puede ser muy profundo e irreconciliable:

"Si los antagonismos son irreductibles e irreconciliables entre sí, es porque los valores que entrañan afectan al sentido que damos a la vida, es decir, la adhesión profunda a una doctrina que orienta nuestras acciones y nuestra jerarquía de valores, y más generalmente, a la elección de principios últimos que sirven de principios directores a nuestra existencia (Freund, 1995: 126).

Gracias a esta posibilidad, no toda decisión puede ser dejada en manos de una lógica exclusiva de negociación, consenso o traducción de intereses. La demanda por mayores cuotas de participación y cambio en las prioridades de las políticas tecnológicas también se dinamiza gracias a los grupos de resistencia y movilización que aprovechan estos antagonismos por vías alternativas a las oficiales y proponen cambiar las reglas del juego democrático, en especial, la inclusión de los afectados y sus intereses. De todos modos, la posibilidad de incluir determinados intereses está limitada, al menos, a la gestión adecuada del proceso de toma de decisiones (Brekke y Eriksen, 1999: 100).

Hasta aquí propongo que un pre-requisito importante para poder abordar la tecnología en contexto es suponer las prácticas políticas en campos de fuerza aún más extensos que los existentes en la configuración de proyectos tecnológicos. Con esto no estoy sosteniendo ninguna clasificación entre lo natural y lo social, ni entre expertos y legos, sólo estoy considerando que para gestionar las luchas y los conflictos por medio de determinadas soluciones tecnológicas, es decir, una gestión sociotécnica de los conflictos sería prudente tomar en cuenta la escalada conflictiva como uno de los medios más de la política en general. Esto porque, uno de los desafíos -al cual me sumo- es el de comprender cómo determinados patrones en tecnología pueden transitar hacia formas menos dominantes, des-atrincheradas y sostenibles. En esta labor, supongo crucial que algunos de los enmarcados contenciosos organizados por los grupos de resistencia se acoplen a las posibilidades de negociar la tecnología, aunque para esto sería recomendable que las modalidades "viejas" de la política no fuesen desacreditadas por completo. Este esfuerzo lo percibo desatendido en los estudios sociales de la tecnología.

La gestión de la escalada como parte de un proceso socio-técnico configurado desde la resistencia, rescata las aportaciones en torno a la gestión y la mediación de conflictos. A su vez, una gestión socio-técnica que tome en cuenta la multi-focalidad del conflicto no podría excluir de su seno a los conflictos explosivos relacionados con determinadas manifestaciones de su escalada violenta y hostil. En este entendido, Galtung critica a aquellas aproximaciones que reducen la gestión del conflicto 
exclusivamente a aquellos conflictos entre pares y a la posibilidad de mantener o eliminar completamente su violencia. Esta forma exclusiva de comprender la gestión de los conflictos excluye a una variedad importante de dinámicas conflictivas, mediante las cuales también se busca crearlos, aumentando la escalada, especialmente cuando éstas no se llevan a cabo entre pares. Con base en estas dos ideas, la gestión del conflicto podría conducirse hacia la escalada, la manutención o la desescalada del conflicto.

Esta gestión de las intensidades conflictivas no proviene sólo del gobierno, es decir, la gobernabilidad de los conflictos también corre por parte de los resistentes, cuando deciden aumentar o escalar la intensidad de sus movilizaciones. Esta sería otra forma de concebir el gobierno multi-actor de los conflictos. Por ejemplo, los grupos movilizados, preocupados 0 interesados consideran distintos medios gracias a los cuales las acciones contenciosas, al escalar su nivel de intensidad, transforman los marcos interpretativos con respecto a un determinado proyecto ${ }^{10}$.

\section{Discusión final: Por una gobernanza menos ingenua}

Aunque el problema de la gobernanza tecnológica o la gobernanza en ciencia y tecnología es amplísimo, me concentraré en una configuración social especial: la configuración movilizada de la tecnología. Mi elección se interesa en demostrar la necesidad de enriquecer la comprensión de este tipo de gobernanza en tecnología, de una forma menos ingenua, rescatando en esta oportunidad, la habilidad para gestionar conflictos desde todos los frentes.

Para enfatizar la importancia de esta perspectiva de gobernanza "desde abajo" expondré algunos resultados del estudio europeo sobre gobernanza en ciencia y tecnología STAGE, el cual propuso una categoría con seis

\footnotetext{
${ }^{10}$ Es conocido en las teorías sobre la gestión de conflictos como el triángulo conflictivo de Galtung (Galtung, 1971).
}

tipos de gobernanza en ciencia y tecnología - una de ellas atinente con la intervención de grupos movilizados posibles de escalar en violencia- la cual se denominó "gobernanza agónica", la que corresponde a una:

[...] gobernanza que toma lugar bajo condiciones de adversidad, y enfrentamiento entre posiciones polarizadas. En este tipo de gobernanza las apuestas son altas, los compromisos no son fáciles de encontrar, porque estas condiciones dificultan el debate y la negociación. Las acciones directas, los boicots, las demostraciones son partes importantes de este proceso. Esto indica los límites de las aproximaciones interactivas-deliberativas, donde el diálogo para la búsqueda no hegemónica y las soluciones comunes es obstaculizado. Al mismo tiempo, las expresiones de la "voz pública" no necesariamente transgreden los bordes de las formas aceptables de la acción política en democracia. Es aquí donde los procesos deliberativos pueden ser poco efectivos, al menos en el esfuerzo por enrolar un indeciso "público" en contra de oponentes los cuales no estarán particularmente susceptibles a cambiar sus puntos de vista.

Continúan sus autores:

En las formas de gobernanza agónicas los principales eventos y recursos de entrada se sitúan en, y son asignados a la arena pública, y con cierta amplitud éstos pueden ser adaptados a las constricciones instaladas por las políticas estatales. Así, el foco está puesto en los esfuerzos por asignar y cambiar el marco dado y sus enmarcados dominantes, concebidos como marcos estructurados inadecuadamente en los términos de las preocupaciones claves de los actores marginados hasta ahora [...] La participación agónica re-configura el alcance y la responsabilidad de las decisiones políticas de Estado, como contrarias, por ejemplo, a procesos de privatización y desregulación (Hagendijk et al, 2005 pág. 180). 
Como ejemplos de este tipo de gobernanza el proyecto STAGE destacó: i) la parcialidad y la escasez de acciones conducentes a la deliberación a través de Europa; ii) la conflictividad en los distintos casos, y iii) la fragilidad de las iniciativas para la participación europea. En parte, en este estudio se planteó que lo que mantiene vivos a los ejercicios de participación son sus elementos agónicos (polémicos), el grado de confusión entre los diferentes modalidades y la constante amenaza del colapso (Hagendijk et al, 2005, pág. 25). Sus autores expusieron que quizás fuese esto precisamente lo que capacita a los ejercicios participativos para invocar (y provocar) cambios -aun cuando las promesas de deliberación pueden nunca ser logradas como tales-.

En Estados Unidos el resultado del actuar de las "bestias" sistémicas lo denuncian constantemente los "movimientos de oposición industrial" y los "movimientos orientados hacia los productos y los procesos tecnológicos" (Hess, 2007), los cuales mediante sus acciones contenciosas luchan por configurar y re-configurar la tecnología, ya sea entregando nuevos significados, o negociando mundialmente otras opciones tecnológicas (Smith, 2005).

En estas dos últimas modalidades se coincide en el esfuerzo de articular la tecnología como una práctica inherentemente política;

aquí las personas luchan contra la dominación presente en la tecnología en general, mientras que en la negociación, los grupos destacan el rol de las controversias sociales para deliberar, y lograr así re-direccionar la tecnología. Como ejemplos de este último tipo están:

El grupo de familiares preocupados por la enfermedad de la distrofia cerebral, el que gracias a su descontento lograron con su movilización social, impulsar líneas de investigación, las cuales desembocaron en la identificación de un gen responsable, el cual transformó en tratables a este tipo de pacientes (Callon \& Rabeharisoa, 2008). Los grupos medioambientales como greenpeace han impulsado los sistemas de refrigeración libres de CFC, así como tecnologías energéticas diversas de origen renovable. La asociación entre grupos pacifistas y antinucleares ha generado todo un saber hacer legal y de boicot a grandes empresas, el cual ha logrado desarrollar un campo de diseño y desarrollo en tecnologías de generación y regulación de energías distribuidas -off grid generation- y microgeneración. La discusión en torno al uso de pesticidas y los Organismos Genéticamente Modificados (OGM) han orientado el interés empresarial en áreas como la agricultura y la alimentación orgánica. Los movimientos que cuestionan la contaminación industrial estadounidense han logrado no sólo ganar moratorias para la ubicación de empresas con criterios de justicia medioambiental, sino que también han influido indirectamente en la institucionalización de una "huella verde" en las empresas, por ejemplo, en el uso de sus residuos (Hess, 2007).

Estos grupos de movilización buscan difuminar los límites de las barreras disciplinarias o de pericia tecnológica y con ello trabajan por crear a partir de la oposición, es por esto que los objetivos prácticos de transformación concreta plateadas por una perspectiva crítica de la tecnología entrega otros elementos, más subjetivos y enriquecedores del aprendizaje contencioso en acción.

La comprensión de las tecnologías deseadas desde la resistencia, considera que determinados procesos de gobernanza tecnológica son intolerables. En este sentido; si se partiese de una actitud más inconformista hacia el análisis de los procesos de co-construcción tecnológica, no sólo ciertas estructuras envolventes podrían interpretarse como opresivas, sino que también determinadas prácticas tecnológicas podrían resultar siendo inaguantables. Esta actitud y comportamiento llega a puertos negociados 0 consensuados en tecnología, siempre y cuando, no sea evadida, en general, la capacidad humana de disentir, e incluso la posibilidad de incrementar la intensidad y hostilidad de las demostraciones de esta capacidad. 
Esta alerta viene siendo recurrente en los estudios sobre gobernanza en ciencia y tecnología. Por ejemplo, en el proyecto STAGE se llama a los estudios en ciencia y tecnología para que sean cuidadosos y menos simplistas a la hora de describir un cambio de modelo en participación, por ejemplo, desde el modelo de expertos al modelo deliberativo. Estos autores llaman la atención sobre lo complejo de los modos de gobierno nacional, lo que aún hace dificultoso proponer generalizaciones entre tecnologías y participación europea en general. Asimismo critican a los modelos deliberativos porque asumen de forma aproblemática las clausuras como formas de consenso social ${ }^{11}$.

En esta línea, Smith \& Stirling reconocen también que en el encuentro de estudios en torno a la tecnología y estudios sobre gobernanza han habido falencias. Los autores identifican coincidencias entre estudiosos de la gestión de las transiciones tecnológicas y los estudiosos de los sistemas ecológicos. Para ambos la transformación de los momenta tecnológicos hacia formas más sustentables depende en gran medida de cómo se modifica y es afectada la elasticidad de los sistemas socio-ecológicos. Ahora bien, aunque la comprensión de esta elasticidad requiere seguir siendo especificada, también es cierto que los análisis en torno a las transiciones socio-técnicas han sido pobres a la hora de proveer modelos heurísticos y herramientas para actores contendientes múltiples, y aquí incluyen a la sociedad civil del lado de los empresarios y el gobierno.

Para Smith \& Stirling la gobernanza reflexiva es una especie de práctica activada desde una base cotidiana por los grupos y movimientos sociales quienes trabajan para que sus prioridades socio-ecológicas sean oídas por las autoridades políticas y los poderes económicos,

\footnotetext{
${ }^{11}$ Problema que coincide con las críticas a la aproximación CST.
}

del mismo modo como lo hacen quienes innovan para ofrecer soluciones que luego adaptan y son adaptadas (Smith \& Stirling, 2008, págs. 21 y 22 [trad. personal]).

Hasta aquí me adhiero al llamado de atención de estos autores sobre este tipo de olvidos en sus descripciones en torno a los estudios de gobernanza en tecnología. Esto porque creo que mientras más plural y discutidos puedan ser los proyectos tecnológicos, las prácticas tecnológicas tendrán más posibilidades de plantearse en términos de calidad de vida, medio ambiente y justicia. En este sentido, no me parece ni justo ni beneficioso relegar la entrada - o considerar indirectamente- en el "parlamento de las cosas" a los ciudadanos preocupados, amenazados por proyectos tecnológicos y a los movimientos sociales, sean éstos nuevos o viejos.

Apoyo la idea de que la gobernanza en sí de la tecnología, en su versión constructivista (Rip et al, 1995) continúe merodeando su giro reflexivo. En este sentido, las palabras de Bourdieu recuerdan que también son las categorías impensadas del pensamiento las que influyen en los aspectos conscientes del pensamiento y la acción; por esta razón para Bourdieu resulta crucial el trabajo teórico y académico, porque sería por medio de éste desde donde es posible sacar a la luz estas categorías, capaces de transformar-transformándose en la acción teórico-prácticasocial (Bourdieu \& Waquart, 2005, cap. 1). La reflexión en acción de Bourdieu recuerda los términos en que las personas no sólo pueden ser consideradas como personas, agencias y entramados movidos por el aprendizaje en los procesos de participación en ciencia y tecnología, sino que también como personas que buscan, reclaman, gritan con sus liderazgos diversos, su derecho a quejarse, dirimir, y si fuese posible, intervenir en las decisiones en ciencia y tecnología.

Para finalizar esta crítica con una consideración esperanzadora, rescato la idea de que los movimientos reivindicativos de la sociedad civil global en los intersticios creados por los dominios translegales (Beck, 2004) favorecen un reconocimiento del poder de los grupos a 
cargo de orquestar polémicas (López Cerezo \& González, 2002), porque entre otras razones, éstos continúan trabajando desde una mirada reflexiva su posición de víctimas (Appadurai, 2002). Reconozco con esto que aún queda mucho por indagar sobre esta mirada renovada de los conflictos, vistos como espacios de posibilidad para construir tecnologías configuradas por las controversias, discusiones y preocupaciones ciudadanas.

\section{Conclusión}

En el siguiente trabajo califiqué de ingenuas a determinadas perspectivas constructivistas en tecnología, las cuales se refirieron a la gobernanza en tecnología. El calificativo de perspectivas ingenuas provino de un trabajo previo que realicé y en el que propuse que las prácticas conflictivas son consideradas parcialmente desde estos enfoques constructivistas. Para esto introduje los enfoques de la Construcción Social de la Tecnología influyentes, para la perspectiva de la Evaluación Constructiva de la Tecnología (ECT), esta última abocada a la formulación y diseño de políticas públicas en ciencia y tecnología.

En segundo término, expuse el enfoque historicista de los Sistemas Socio-Técnicos de Gran Escala (SSTGE), el que ligué con la idea de los artefactos vistos como "suaves bestias" que requieren ser dirigidas, gobernando sus conflictos internos con el fin de que sean coherentes con su sentido evolutivo -momentum-. Aquí, la gestión de conflictos la ubiqué en organizaciones, instituciones, e incluso gobiernos más represores que tolerantes a las resistencias de su entorno. En particular, me referí a los gobiernos proclives a activar el silencio de los conflictos a través de las modalidades tradicionales de la aplicación de la ley y el orden. Con esto introduje la pertinencia de los planteamientos de Galtung sobre la gestión de conflictos, específicamente la gestión de la intensidad hostil provenientes de los grupos resistentes y afectados.

Para finalizar expuse algunas críticas a las propuestas de gobernanza en tecnología con respecto a su interpretación en torno a la posibilidad de participar socio-técnicamente desde la resistencia. Con todo esto propuse que una noción de gobernanza en tecnología menos ingenua, sería aquella que contemplase un rol más protagónico para las estrategias y mecanismos activados desde los grupos y movilizaciones ciudadanas en el contexto global.

\section{Bibliografía}

Appadurai, A. 2002. "Deep Democracy: Urban Governmentality and the Horizion of Politics," Environment and Urbanization 13 (2). 23-43. October 2001.

Baigorrotegui, G. 2008. "Dinámica y Gestión Social de las Electroenergías. Abordando su dimensión controversial". Tesis Doctoral. San Sebastián. Universidad del País Vasco/Euskal Herriko Unibersitatea.

Beck, U. 2004. Poder y Contrapoder en la era global. Barcelona, Paidós.

Bloor, D. 1976. Knowledge and Social Imagery. London \& Boston MA: Routledge \& Kegan Paul.

Bourdieu, P. \& Wacquart, L. 2005 Una Invitación a la Sociología Reflexiva. Coyoacán, Siglo veintiuno.

Brekke, 0. y Eriksen, E. 1999. "Technology Assessment in a deliberative perspective" VON SCHOMBERG, R. (ed.), Democratising Technology. Theory and Practice of Deliberative Technology Policy. 93-119. Hengelo, National Centre for Human and Public Affairs.

Brown, S. y Lee, N. 1998. "La alteridad y el Actor-Red. El continente no descubierto", en Domènech, $\mathrm{M}$. y Tirado, F. J. (comp.), Sociología Simétrica. 219-248. Barcelona, Gedisa.

Callon, M. 1995. "Algunos elementos para una sociología de la traducción: la domesticación de las vieiras y los pescadores de la bahía de St. Brieuc" en Iranzo, J. M. et al (Comp.) Sociología de la ciencia y de la tecnología. 259-82. Madrid, CSIC. 
Callon, M. y Rabeharisoa, V. 2008. "The growing engagement of emergent concerned groups in political and economic life. Lessons from the french Association of Neuromuscular Disease Patients". Science, Technology and Human Values (33/2), 230-261.

Collins, H. M y Yearley, S. 1992. "Epistemological chicken", en Pickering, A. Science as Practice and Culture. 30126. Londres, The University of Chicago Press.

Entelman, R. 2002. Teoría de Conflictos. Hacia un Nuevo Paradigma. Barcelona, Gedisa.

Galtung, J 1971. "Peace Thinking", en Lepawski, A.; Buehring, E.; Lasswell, H. (ed.). The Search for World Order. New York, Meredith.

Hacking, I. 2001. ¿La Construcción Social de Qué? Barcelona, Paidós.

Hagendijk, R.; Healey, P.; Horst, M.; Irwin, A. 2005. Science, Technology and Governance in Europe. Challenges of Public Engagement. Final Report, Stage Project.

Hård, M. 1993. "Beyond Harmony and Consensus: a Social Conflict Approach to Technology", Science, Technology, and Human Values (18/4), 408-432.

Hughes, T. P. 1983. Networks of Power. London, Johns Hopkins University Press.

Hughes, T. P. 1987. "The Evolution of Large Technological Systems", en Bijker, W.; Hughes, T.P.; Pinch, T. (ed.). The Social Construction of Technological Systems. New Directions in the Sociology and History of Technoloy. 51-82. Cambridge MIT Press, 1990.

Jæger, B. 2001. "Strengths and Weaknesses of Constructivistic Studies of Technology", Institute for Samfundsvidensab og Erhveervsokonomi. Documento de trabajo (18/01).(http://www.ruc.dk/upload/ application/pdf/f51d6748/18-01.pdf (13/03/05)).
Law, J. 1987. "The Anatomy of a Socio-technical Struggle: The Design of the TSR2" en Bijker, W.; Hughes, T.P.Y Pinch, T. (ed.), The Social Construction of Technological Systems. New Directions in the Sociology and History of Technoloy. 44-69. Cambridge MIT Press, 1990.

López Cerezo, J. A. y González, M. I. 2002. Políticas del Bosque. Expertos, políticos y Ciudadanos en la Polémica del Eucalipto en Asturias. Madrid, Cambridge University Press.

Martin, B. 2006. "Paths to social change: conventional politics, violence and non-violence", en Summy, R. (ed.). Nonviolent Alternatives for Social Change. Oxford: Eolss Publishers. (http://www.uow.edu.au/arts/sts/ bmartin/pubs/06eolss.html (14/02/06)).

Pinch, T. J. y Bijker, W.. 1984. "The Social Construction of Facts and Artefacts: or How the Sociology of Science and the Sociology of Technology might Benefit Each Other", Social Studies of Science (14), 399-441.

Rip, A.; Misa, T. J.; Schot, J. 1995. "Constructive Technology Assessment: a new paradigm for managing technology in society", en Rip, A.; Misa, T. J.; Schot, J. (ed.). Managing Technology in Society: The Approach of Constructive Technology Assessment. 1-14. London, Pinter.

Rip, A. 1994. "Science and Technology Studies and Constructive Technology Assessment”, European Association for the Study of Science and Technology EASST Review (13/3).

Rip, A. 2002. "Co-evolution of science, technology and society", Revisión experta del Bundesministerium Bildung. (http://www.sciencepolicystudies.de/dok/ expertise-rip.pdf (15/09/08)).

Smith, A. y Stirling, A. 2006. "Inside or out? open or closed? positioning the governance of sustainable technology", SPRU. Documentos de Trabajo (148). (http://www. sussex.ac.uk/spru/1-6-1-2-1.html (20/02/08)). 
Smith, A. \& Stirling, A. 2008. "Social-ecological resilience and socio-technical transitions: critical issues for sustainability governance", STEPs working paper (8). Brighton, Spru.

Snow, D. Y Benford, R. 2000. "Framing processes and social movements: an overview and assessment", Annual Rev. Sociology (26), 611-39.

Stoker, G 1998. "Governance as theory: five propositions", International Social Science Journal (50/155), 17-28.

Williams, R. y Edge, D. 1996. "The social shaping of technology”, Research Policy (25), 856-899.

Winner, L. 1993. "Opening the black box and finding it empty: Social constructivism and the philosophy of technology", Science, Technology and Human Values (18), 362-78. 


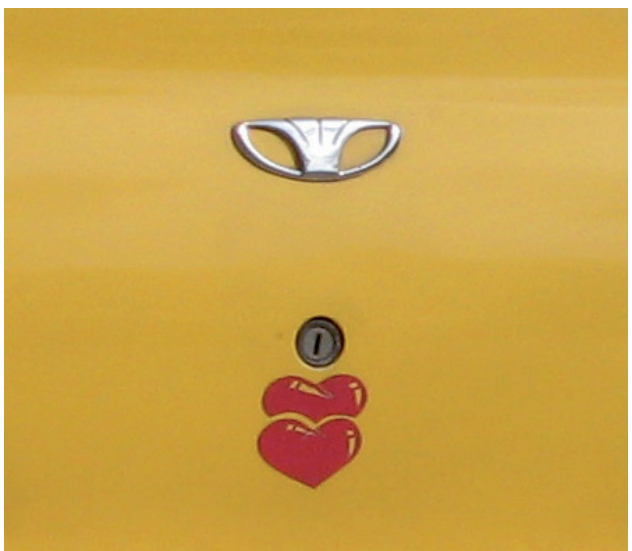

¡MECANIZARSE!

iTrrrum

Trrrum

Trrrum!

iTrac triki tac

mecanizarme quiero!

iDe mi cerebro, de mi carne, de mi esqueleto procede este afán!

iMe vuelvo loco

por subir

sobre las dinamos!

Mi lengua húmeda lame los cables de cobre.

iEn mis venas,

las vagonetas accionadas a mano

persiguen las locomotoras!

iTrrrum

Trrrum

Trrrum

iTrac triki tac

mecanizarme quiero!

Encontraré sin duda una salida.

iÚnicamente seré feliz

el día que consiga

encajar una turbina en mi vientre

y colocar una doble hélice a mi rabo!

iTrrrum

Trrrum

Trrrum

iTrac triki tac

mecanizarme quiero!

\section{Nazim Hikmet}

